\title{
An Arithmetic Reduction of Finite Rank 3 Geometries with Linear Spaces as Plane Residues and with Dual Linear Spaces as Point Residues
}

\author{
CÉCILE HUYBRECHTS* \\ huyb@ulb.ac.be \\ Département de Mathématique, C.P.216 Université Libre de Bruxelles, Campus Plaine 1050 Bruxelles, Belgium
}

Received December 17, 1993; Revised May 19, 1995

\begin{abstract}
Let $\Gamma$ be a rank three incidence geometry of points, lines and planes whose planes are linear spaces and whose point residues are dual linear spaces (notice that we do not require anything on the line residues). We assume that the residual linear spaces of $\Gamma$ belong to a natural class of finite linear spaces, namely those linear spaces whose full automorphism group acts flag-transitively and whose orders are polynomial functions of some prime number. This class consists of six families of linear spaces. In $\Gamma$ the amalgamation of two such linear spaces imposes an equality on their orders leading, in particular, to a series of diophantine equations, the solutions of which provide a reduction theorem on the possible amalgams of linear spaces that can occur in $\Gamma$.
\end{abstract}

We prove that one of the following holds (up to a permutation of the words "point" and "plane").

A) the planes of $\Gamma$ and the dual of the point residues belong to the same family and have the same orders,

B) the diagram of $\Gamma$ is in one of six families,

C) the diagram of $\Gamma$ belongs to a list of seven sporadic cases.

Finally, we consider the particular case where the line residues of $\Gamma$ are generalized digons.

Keywords: incidence geometry, finite linear space, parameters

\section{Introduction}

We assume some knowledge of a few basic definitions and facts from the theory of diagram geometries (see for instance [2] or [3]). We introduce a few definitions and notation. Let $\Gamma$ be a geometry over $I=\{0, \ldots, k\}$ and let $i \in I$. As usual, we say that $\Gamma$ has orders $\left(q_{0}, \ldots, q_{k}\right)$ if for each $i$ in $I$, there is a positive integer $q_{i}$ such that for each flag $F$ of cotype $i$, the number of $i$-elements in the residue of $F$ is $q_{i}+1$. The number $q_{i}$ is called the $i$-order of $\Gamma$. We recall that a linear space is a rank 2 geometry consisting of points and lines such that any two points are incident with exactly one line, any point is incident with at least two lines and any line with at least two points. From now on, we will use "linear space" for "finite linear space". 
We will consider geometries belonging to

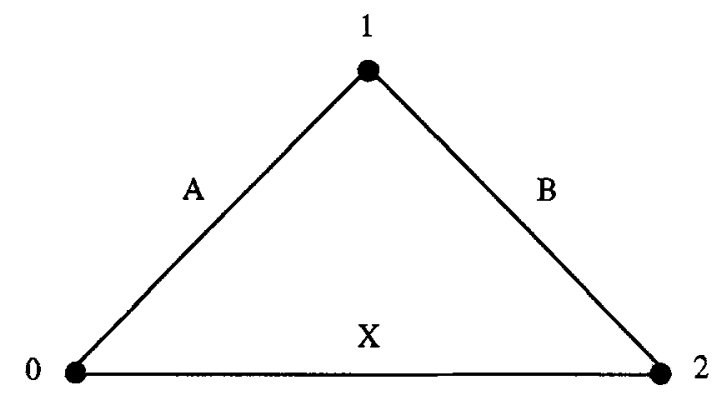

where $A$ is a class of linear spaces and $B$ is a class of dual linear spaces. Since the class $X$ is not important for our work, we denote the above diagram by [ $A \triangle B$ ], where the symbol $\Delta$ indicates that this diagram is triangular. If we want to specify that $X$ is a class of generalized digons, then we write $[A \cdot B]$ instead of $[A \triangle B]$. We say that a geometry $\Gamma$ over $\{i, j\}(i<j \in \mathbb{N})$ belongs to $[L]$ (resp. $\left.\left[L^{*}\right]\right)$ if $\Gamma$ is a linear space whose points are the $i$-elements (resp. $j$-elements) and whose lines are the $j$-elements (resp. $i$-elements). The diagram $\left[L^{*}\right]$ is the dual diagram of $[L]$. Let $[Y]$ denote a diagram. From now on, we say that " $\Gamma$ is a $[Y]$-geometry" instead of " $\Gamma$ is a geometry belonging to $[Y]$ ". We also write $\Gamma \in[Y]$.

Let $\Gamma$ be a $\left[L \Delta L^{*}\right]$-geometry having orders. We make assumptions on $\Gamma$ motivated by the classification of linear spaces having a flag-transitive group of automorphisms [4]. The flag-transitive linear spaces (i.e. those having a flag-transitive group of automorphisms) are known except if the group involved is a 1-dimensional affine group [4]. We take from [4] the orders of the known thick flag-transitive linear spaces and we do not require more data on those. Then we look at the conditions imposed by the orders of these linear spaces. Indeed, if we "amalgamate" two classes $A$ and $B$ of linear spaces of respective orders $(a, b)$ and $(c, d)$ to get a diagram $\left[A \Delta B^{*}\right]$, the numbers $b$ and $d$ must be equal. The main part of this article consists in studying the diophantine equations resulting from " $b=d$ ". We deduce from this study a reduction theorem on $\left[L \Delta L^{*}\right]$-geometries. Next, we examine the particular case where the line residues are generalized digons: we use the Doyen-Hubaut theorem [6] to eliminate some diagrams containing a residual projective plane and for most of the remaining diagrams $\Delta$, we give a geometry belonging to $\Delta$.

No knowledge of linear spaces or of group theory is needed for this paper. The only consequence of the group-theoretic assumption is the restriction of the class of the residual linear spaces of the geometries we consider. The method used here is very general; it may be applied to every amalgamation problem of rank two geometries having orders and it has indeed been applied in such work by various authors.

Let $K$ be the class of rank 3 geometries whose residues are either generalized digons or linear spaces. Can we classify those geometries belonging to $K$ whose residual linear spaces belong to the family of known flag-transitive linear spaces? This is not an easy question. This paper can be considered as a first and elementary step in this direction or towards the classification of flag-transitive geometries belonging to $K$.

The author is grateful to Buekenhout and to Doyen for helpful conversations. 


\section{Main results}

We give a specific diagram to some classes of flag-transitive linear spaces according to their orders (see [4] for more information). We distinguish six families of diagrams: we first write the notation for the diagram, next the corresponding class of linear spaces. We also specify the orders of these linear spaces and if necessary some restrictions. In all this work, these six families of diagrams may be viewed as families of diagrams with given orders. Here $q$ denotes a prime power.

1) $\left[P_{n}(q)\right]$ Projective Spaces $\left(q, q^{n-1}+\cdots+q\right), n \geq 2$,

2) $\left[A_{n}^{f}(q)\right]$ Affine Spaces $\left(q-1, q^{n-1}+\cdots+q\right), n \geq 2$,

3) $[W(q)]$ Witt-Bose-Shrikhande Spaces $(q / 2-1, q), q=2^{s}, s \geq 3$,

4) $[$ Her] Hering Spaces $(8,90)$,

5) $\left[U_{H}(q)\right]$ Hermitian Unitals $\left(q, q^{2}-1\right), q \geq 3$,

6) $\left[U_{R}(q)\right]$ Ree Unitals $\left(q, q^{2}-1\right), q=3^{2 r+1}, r \geq 1$.

The Ree Unitals $U_{R}\left(3^{2 r+1}\right)$ are in fact defined for $r=0$. However, as $U_{R}(3) \simeq W(8)$, we only consider the case where $r \geq 1$. Similarly, we assume that $q \geq 3$ for the Hermitian Unitals $U_{H}(q)$ because $U_{H}(2)$ is an affine plane.

From the classification of finite flag-transitive linear spaces $\Gamma$ [4], we know that either $\Gamma$ belongs to one of the above six families, or $\Gamma$ is a complete graph, or the full automorphism group of $\Gamma$ is a one-dimensional affine group. However, we do not consider the two latter in this paper, because their orders can take too general values.

Here also we distinguish $\left[P_{n}(q)\right]$ and $\left[P_{n}^{*}(q)\right], \ldots$, according to the choice of points and lines. We denote by $\Sigma$ the union of the above six families of diagrams and by $\Sigma^{*}$ the set of the dual diagrams of $\Sigma$. We say that a $[A \Delta B]$-geometry $\Gamma$ is $\Sigma$-homogeneous if for each $i$ in $\{0,2\}$, there is a diagram of $\Sigma \cup \Sigma^{*}$ such that the residues of the $i$-elements of $\Gamma$ belong to this diagram. In this case, we denote the residue of an $i$-element by $\Gamma_{l}$.

Theorem 2.1 Let $\Gamma$ be a $\Sigma$-homogeneous [ $\left.L \Delta L^{*}\right]$-geometry. Then $\Gamma$ belongs, up to a permutation of the types 0 and 2, to one of the following diagrams.

A) $[A \Delta B]$, where $A=B^{*} \in\left\{P_{n}(q), A_{n}^{f}(q), W(q), \operatorname{Her}, U_{H}(q), U_{R}(q)\right\}$

B) six families

1. $\left[P_{n}(q) \Delta P_{m}^{*}\left(q^{\prime}\right)\right]$ with $q^{n-1}+\cdots+q^{2}+q=q^{\prime m-1}+\cdots+q^{\prime 2}+q^{\prime}, n \neq m$ and $n, m \neq 2$.

2. $\left[A_{n}^{f}(q) \triangle A_{m}^{f *}\left(q^{\prime}\right)\right]$ with $q^{n-1}+\cdots+q^{2}+q=q^{\prime m-1}+\cdots+q^{2}+q^{\prime}, n \neq m$ and $n, m \neq 2$.

3. $\left[P_{n}(q) \Delta A_{m}^{f *}\left(q^{\prime}\right)\right]$ with $q^{n-1}+\cdots+q^{2}+q=q^{\prime m-1}+\cdots+q^{2}+q^{\prime}$ (moreover, if either $n=2$ or $m=2$, then $m=n$ and $q=q^{\prime}$ ).

4. $\left[W\left(2^{s}\right) \triangle A_{2}^{f *}\left(2^{s}\right)\right]$

5. $\left[W\left(2^{s}\right) \triangle P_{2}^{*}\left(2^{s}\right)\right]$

6. $\left[U_{R}\left(3^{2 r+1}\right) \triangle U_{H}^{*}\left(3^{2 r+1}\right)\right]$

C) seven sporadic cases

1. $\left[U_{H}(3) \triangle P_{2}^{*}(8)\right]$

2. $\left[U_{H}(3) \Delta A_{2}^{f *}(8)\right]$ 
3. $\left[U_{H}(3) \Delta W^{*}(8)\right]$

4. $\left[U_{H}(11) \triangle P_{5}^{*}(3)\right]$

5. $\left[U_{H}(11) \triangle A_{5}^{f *}(3)\right]$

6. [Her $\left.\triangle P_{3}^{*}(9)\right]$

7. [Her $\left.\triangle A_{3}^{f *}(9)\right]$

Theorem 2.2 Let $\Gamma$ be a $\Sigma$-homogeneous $\left[L \cdot L^{*}\right]$-geometry. Then $\Gamma$ belongs, up to a permutation of the types 0 and 2, to one of the diagrams of Theorem 2.1, where " $\Delta$ " is replaced by "." and from which we remove cases B)5 and C) 1 .

Under the additional assumption that $\Gamma$ admits a flag-transitive automorphism group, Theorem 2.1 can be improved as follows: "Either $\Gamma_{0} \simeq \Gamma_{2}$ or the diagram of $\Gamma$ is given by case B)1 or B)3, with $(n, q)-\left(m, q^{\prime}\right)$ in the latter case" [7].

Let us mention that it is possible to deduce other reduction theorems from Theorem 2.1 by modifying the classes of geometries corresponding to the diagrams of $\Sigma$ because to prove Theorem 2.1 , the only information we need on the geometries $\Gamma$ we consider is the polynomial function defining the 1-orders of $\Gamma_{0}$ and $\Gamma_{2}$.

\section{A few results from number theory}

This section contains one proposition about diophantine equations and some easy facts needed afterwards.

Proposition 3.1 [8] If $q$ is a prime power and $y$ is a natural number, then the only solutions of the equation $q^{n}+\cdots+q^{2}+q+1=y^{2}$ are:

(1) $n=1, q=3$ and $y=2$,

(2) $n=1, q=8$ and $y=3$,

(3) $n=3, q=7$ and $y=20$,

(4) $n=4, q=3$ and $y=11$.

From now on $q, q^{\prime}$ denote prime powers, and $n, m$ are integers with $n, m \geq 2$.

Fact 3.2 Assume that $q^{n-1}+\cdots+q^{2}+q=q^{\prime m-1}+\cdots+q^{\prime 2}+q^{\prime}$. Then $q=q^{\prime}$ if and only if $n=m$, and $n=2$ if and only if $m=2$.

Proof: The proof is straightforward.

Fact 3.3 The unique solution of the equation $q^{n-1}+\cdots+q^{2}+q=90$ is given by $q=9$ and $n=3$.

Proof: Observe first that $q$ divides 90 . As $q$ is a prime power, $q=2,3,5$ or 9. Moreover, $q^{n-1}+\cdots+q^{2}+q=90$ if and only if $\left(q^{n}-1\right)=91(q-1)$. We immediately deduce the result. 


\section{The Doyen-Hubaut theorem}

Here we write in terms of diagrams and without giving all details, the Doyen-Hubaut theorem [6].

Proposition 4.1 [6] Let $\Gamma$ be a geometry belonging to $\left[L \cdot P_{2}(q)\right]$ with finite orders $(s, t, t)$. Then one of the following holds:

(1) $\Gamma \in\left[A_{2}^{f}(q) \cdot P_{2}(q)\right]$

(2) $\Gamma \in\left[P_{2}(q) \cdot P_{2}(q)\right]$

(3) $t=(s+1)^{2}$

(4) $t=(s+1)^{3}+(s+1)$

We immediately deduce the following statement.

Proposition 4.2 Let $\Gamma$ be $a\left[L \cdot P_{2}(q)\right]$-geometry oforders $(a, b, c)$. If $m \geq 3$ then $(a, b, c)$ is not equal to $\left(2^{m-1}-1,2^{m}, 2^{m}\right)$.

\section{Proof of the Theorems}

Let $\Gamma$ be a $\Sigma$-homogeneous [ $L \triangle L^{*}$ ]-geometry. Let $a$ (resp. $b$ ) be the order of the 0 -elements (resp. 1-elements) of $\Gamma_{2}$ and let $c$ (resp. $d$ ) be the order of the 2-elements (resp. 1-elements) of $\Gamma_{0}$. By definition of the orders of a geometry, we have $b=d$.

We now look at the diophantine equations resulting from the equality " $b=d$ ". Since $P_{n}(q), A_{n}^{f}(q), W(q)$ and Her have a line order equal to $q^{n-1}+\cdots+q^{2}+q$ (with $n=2$ and $(n, q)=(3,9)$ respectively for the two latter cases) and $U_{H}(q), U_{R}(q)$ have a line order equal to $q^{2}-1$, all the equations we obtain are particular cases of one of the following equations: $q^{n-1}+\cdots+q^{2}+q=q^{2}-1, q^{2}-1=q^{2}-1$ and $q^{n-1}+\cdots+q^{2}+q=q^{\prime m-1}+\cdots+q^{\prime 2}+q^{\prime}$.

By Proposition 3.1, the only solutions of the equation $q^{n-1}+\cdots+q^{2}+q=q^{2}-1$, where $q, q^{\prime}$ are prime powers with $q^{\prime} \geq 3$ and $n$ is an integer $\geq 2$, are $\left(n, q, q^{\prime}\right)=(2,8,3)$ and $(5,3,11)$. Clearly $q^{2}-1=q^{\prime 2}-1$ implies that $q=q^{\prime}$. It suffices then to use Facts 3.2 and 3.3 to end the proof of Theorem 2.1 .

By Proposition 4.2 , there does not exist a geometry belonging to $\left[W\left(2^{\prime \prime}\right) \cdot P_{2}^{*}\left(2^{\prime}\right)\right]$ or $\left[U_{H}(3) \cdot P_{2}^{*}(8)\right]$. Consequently, using Theorem 2.1, we deduce Theorem 2.2.

Remark We get above the equation $q^{n-1}+\cdots+q^{2}+q=q^{\prime m-1}+\cdots+q^{2}+q^{\prime}$ where $q, q^{\prime}$ are prime powers and $n, m$ are integers $\geq 2$. One non-trivial solution (that is with $q \neq q^{\prime}$ and $\left.n \neq m\right)$ is known and is given by $\left(n, q, m, q^{\prime}\right)=(3,5,5,2)$ [9]. We do not know how many non-trivial solutions there are. This problem seems to be unsolved (see [9] for more information on this equation).

\section{Examples}

Let $\Delta$ be a diagram of Theorem 2.2. In this section, we discuss the existence of a geometry belonging to $\Delta$. If we know the existence of geometries on $\Delta$, we only give one example (see [7] for references to more examples). Let $\Gamma$ be a $D_{n}$-geometry over $\mathbb{F}_{q}, n \geq 3$. Denote 
by $\{1, \ldots, n\}$ the set of types of $\Gamma$. The $\{n-2, n-1, n\}$-truncation of $\Gamma$ is a geometry belonging to $\left[P_{n-1}(q) \cdot P_{n-1}^{*}(q)\right]$. Let $\tilde{\Gamma}$ be the half-spin geometry $D_{n, n}$ (we recall that the $n$-elements of $\Gamma$ may be identified with a set of points of a projective space $\mathbb{P}$ of dimension $2_{-1}^{n-1}$ over $\mathbb{F}_{q}$ and that $\Gamma$ may be represented in $\mathbb{P}$; we call $\tilde{\Gamma}$ this representation). It is well-known that one example of $\left[A_{n-1}^{f}(q) \cdot P_{n-1}^{*}(q)\right]$ can be obtained by deleting a suitable projective hyperplane from $\tilde{\Gamma}$ and by truncating on $\{n-2, n-1, n\}$.

We give here a construction due to Pasini [5] which provides a geometry for many diagrams of Theorem 2.2. We say that a linear space $\Gamma$ admits a parallelism if there is an equivalence relation $\|$ defined on the lines of $\Gamma$ such that for every line $L$ of $\Gamma$ and every point $p$ of $\Gamma$, there is a unique line $M$ of $\Gamma$ such that $M$ is incident with $p$ and $M \| L$. The equivalence class of a line $L$ of $\Gamma$ is denoted by $\infty(L)$ and the set of the equivalence classes is denoted by $\infty(\Gamma)$. Notice that $|\infty(\Gamma)|=b+1$ if $\Gamma$ is of orders $(a, b)$. Let $\Gamma$ and $\Gamma^{\prime}$ be two linear spaces of orders $(a, b)$ and $\left(a^{\prime}, b^{\prime}\right)$, respectively. Assume that $\Gamma$ and $\Gamma^{\prime}$ admit a parallelism. If $\infty(\Gamma)$ and $\infty\left(\Gamma^{\prime}\right)$ have the same cardinality (that is if $b=b^{\prime}$ ), we may identify them, and a rank 3 geometry $\tilde{\Gamma}$ can be constructed as follows: the points (resp. planes) of $\tilde{\Gamma}$ are those of $\Gamma$ (resp. $\left.\Gamma^{\prime}\right)$; the lines of $\tilde{\Gamma}$ are the pairs $\left(X, X^{\prime}\right)$ where $X$ is a line of $\Gamma$ and $X^{\prime}$ is a line of $\Gamma^{\prime}$ with the property that $\infty(X) "=" \infty\left(X^{\prime}\right)$; every point of $\tilde{\Gamma}$ is incident with every plane of $\tilde{\Gamma}$; a point (resp. plane) $x$ of $\tilde{\Gamma}$ is incident with a line $\left(X, X^{\prime}\right)$ of $\tilde{\Gamma}$ if $x$ is incident with $X$ in $\Gamma$ (resp. $X^{\prime}$ in $\Gamma^{\prime}$ ). If $\Gamma$ belongs to $[A]$ and $\Gamma^{\prime}$ to $[B]$, it is not difficult to show that $\tilde{\Gamma}$ belongs to $\left[A \cdot B^{*}\right]$.

Any linear space $\Gamma$ belonging to $[\mathrm{Her}],\left[U_{R}(q)\right],[W(q)],\left[U_{H}(q)\right]$ or $\left[A_{n}^{f}(q)\right]$ admits a parallelism [5]. The case $\Gamma \in\left[P_{n}(q)\right]$ is more complicated : if $n$ is even, $\Gamma$ does not admit a parallelism; if $n$ is odd and either $q=2$ or $n=2^{i}-1(i=2,3, \ldots)$, then $\Gamma$ admits a parallelism ([1] or [5]). The general case $n$ odd seems to be unsolved.

Consequently, for any diagram $\left[A \cdot B^{*}\right]$ of the list of Theorem 2.2, where neither $A$ nor $B$ is a class of projective spaces, there is a geometry belonging to $\left[A \cdot B^{*}\right]$. There is moreover a geometry belonging to $\left[\mathrm{Her} \cdot P_{3}^{*}(9)\right],\left[P_{3}(5) \cdot P_{5}^{*}(2)\right],\left[P_{3}(5) \cdot A_{5}^{f *}(2)\right]$ and $\left[A_{3}^{f}(5) \cdot P_{5}^{*}(2)\right]$ (the latter three diagrams correspond to the unique known non-trivial solution $\left(n, q, m, q^{\prime}\right)$ of the equation $\left.q^{n-1}+\cdots+q^{2}+q=q^{\prime m-1}+\cdots+q^{2}+q^{\prime}\right)$.

In conclusion, there is a geometry belonging to any diagram of the list of Theorem 2.2 except for C) 4 and for cases B) 1 and B) 3 provided there exists another non-trivial solution to the equation given above. We do not know if there is a geometry belonging to these latter diagrams.

Remark There are two non-isomorphic linear spaces belonging to [Her] and for some values of $q$, there are Desarguesian and non-Desarguesian affine planes of order $q$. Let [Her ${ }_{1}$ ] and $\left[\mathrm{Her}_{2}\right]$ denote the two Hering's spaces. If $\Gamma$ is a Desarguesian (resp. non-Desarguesian) $\left[A_{2}^{f}(q)\right]$-geometry, we say that $\Gamma$ belongs to $\left[\mathrm{Af}_{D}\right]$ (resp. [Af $\left.\mathrm{AD}_{\mathrm{ND}}\right]$ ). The above Pasini construction yields in fact an example of a geometry $\Gamma$ for any $\Gamma$ belonging to $\left[\mathrm{Her}_{1} \cdot \mathrm{Her}_{1}^{*}\right]$, $\left[\mathrm{Her}_{2} \cdot \mathrm{Her}_{2}^{*}\right],\left[\mathrm{Her}_{1} \cdot \mathrm{Her}_{2}^{*}\right],\left[\mathrm{Af}_{\mathrm{D}} \cdot \mathrm{Af}_{\mathrm{D}}^{*}\right],\left[\mathrm{Af}_{\mathrm{ND}} \cdot \mathrm{Af}_{\mathrm{ND}}^{*}\right],\left[\mathrm{Af}_{\mathrm{D}} \cdot \mathrm{Af}_{\mathrm{ND}}^{*}\right],\left[W\left(2^{s}\right) \cdot \mathrm{Af}_{\mathrm{D}}^{*}\left(2^{s}\right)\right]$, etc.

\section{References}

1. A. Beutelspacher, D. Jungnickel, and S.A. Vanstone, "On the chromatic index of finite projective spaces," Geom. Dedicata 32 (1989), 313-318. 
2. F. Buekenhout, "The basic diagram of a geometry," in Geometries and Groups, Berlin, Lecture Notes in Math. 983, Springer-Verlag, 1981, 1-29.

3. F. Buekenhout, "Foundations of incidence geometry," in Handbook of Incidence Geometry, F. Buekenhout (Ed.), Elsevier, Amsterdam, 1994.

4. F. Buekenhout, A. Delandtsheer, J. Doyen, P.B. Kleidman, M.W. Liebeck, and J. Saxl, "Linear spaces with flag-transitive automorphism groups," Geom. Dedicata 36 (1990), 89-94.

5. F. Buekenhout, C. Huybrechts, and A. Pasini, "Parallelism in diagram geometry," Bull. Belg Math Soc. 3 (1994), 355-397.

6. J. Doyen and X. Hubaut, "Finite regular locally projective spaces," Math. Z. 119 (1971), 83-88.

7. C. Huybrechts, "A reduction of flag-transitive $\left[L \Delta L^{*}\right]$-geometries," in preparation.

8. W. Ljunggren, "Some theorems on indeterminate equations of the form $\left(x^{n}-1\right) /(x-1)=y^{q}$," Norsk Mat. Tidsskr. 25 (1943), 17-20.

9. A. Makowski and A. Schinzel, "Sur l'équation indéterminée de R. Goormaghtigh," Mathests (4) 68 (1959), $128-142$. 